\title{
Synthesis of folate-chitosan nanoparticles loaded with ligustrazine to target folate receptor positive cancer cells
}

\author{
LICHUN CHENG $^{1}$, HUI MA ${ }^{1}$, MINGKUN SHAO ${ }^{1}$, QING FAN ${ }^{1}$, HUIYI LV $^{1}$, JINYONG PENG $^{2}$, TANGNA HAO $^{1}$, \\ DAIWEI LI ${ }^{1}$, CHENYANG ZHAO ${ }^{1}$ and XINGYUE ZONG ${ }^{3}$ \\ ${ }^{1}$ Department of Pharmacy, The Second Affiliated Hospital of Dalian Medical University, Dalian, Liaoning 116027; \\ ${ }^{2}$ College of Pharmacy, Dalian Medical University, Dalian, Liaoning 116044, P.R. China; ${ }^{3}$ Medical Sciences Program, \\ Indiana University School of Medicine, Bloomington, IN 47408, USA
}

Received May 9, 2016; Accepted March 27, 2017

DOI: $10.3892 / \mathrm{mmr} .2017 .6740$

\begin{abstract}
In addition to its vasodilatory effect, ligustrazine (LZ) improves the sensitivity of multidrug resistant cancer cells to chemotherapeutic agents. To enhance the specificity of LZ delivery to tumor cells/tissues, folate-chitosan nanoparticles (FA-CS-NPs) were synthesized by combination of folate ester with the amine group on chitosan to serve as a delivery vehicle for LZ (FA-CS-LZ-NPs). The structure of folate-chitosan and characteristics of FA-CS-LZ-NPs, including its size, encapsulation efficiency, loading capacity and release rates were analyzed. MCF-7 (folate receptor-positive) and A549 (folate receptor-negative) cells cultured with or without folate were treated with FA-CS-LZ-NPs, CS-LZ-NPs or LZ to determine cancer-targeting specificity of FA-CS-LZ-NPs. Fluorescence intensity of intracellular LZ was observed by laser scanning confocal microscopy, and concentration of intracellular LZ was detected by HPLC. The average size of FA-CS-LZ-NPs was $182.7 \pm 0.56 \mathrm{~nm}$, and the encapsulation efficiency and loading capacity was $59.6 \pm 0.23$ and $15.3 \pm 0.16 \%$ respectively. The cumulative release rate was about $95 \%$ at $\mathrm{pH} 5.0$, which was higher than that at $\mathrm{pH}$ 7.4. There was higher intracellular LZ accumulation in MCF-7 than that in A549 cells and intracellular LZ concentration was not high when MCF-7 cells were cultured with folate. These results indicated that the targeting specificity of FA-CS-LZ-NPs was mediated by folate receptor. Therefore, the FA-CS-LZ-NPs may be a potential folate receptor-positive tumor cell targeting drug delivery system that could possibly overcome multidrug resistance during cancer therapy.
\end{abstract}

Correspondence to: Dr Qing Fan, Department of Pharmacy, The Second Affiliated Hospital of Dalian Medical University, 467 Zhongshan Road, Shahekou, Dalian, Liaoning 116027, P.R. China E-mail: fq731@sina.com

Key words: folate receptor, tumor targeting, ligustrazine, nanoparticle

\section{Introduction}

Ligustrazine (LZ), a bioactive component from the traditional Chinese medicine ligusticum, is primarily used in China as a vasodilator (1). In recent years, it has been reported that LZ inhibits tumor metastasis and improves the sensitivity of multidrug resistant tumor cells to chemotherapeutic agents (2). However, LZ is chemically unstable with a half-life of $\sim 1.5 \mathrm{~h}$ (3) and lacks a compatible drug delivery system, which limits its potential as a chemotherapeutic agent in the management of cancer. Our previous study demonstrated that liposomes loaded with $\mathrm{LZ}$ enhanced the effect of $\mathrm{LZ}$ in reversing multi-drug resistance (MDR) in K562/ADM cells (4). However, liposome is not an ideal carrier for anticancer agents due to its low encapsulation efficiency (39.5\%) and lack of active targeting (5). Therefore, the current study synthesized folate-conjugated chitosan nanoparticles (FA-CS-NPs) loaded with LZ to enhance the targeting ability and biocompatibility mediated by folate receptor.

Chitosan NPs are emerging as drug delivery system due to its favorable characteristic features such as size, biocompatibility, high drug encapsulation efficiency, controlled drug release potential and long circulating half-life (6). Furthermore, due to the presence of primary amino groups, CS-NPs are easily modified by various ligands, including folate (7), epidermal growth receptor (8) and polypeptides (9). Thus, modifications of CS-NPs with ligands specific for receptors on tumor cells may enhance the specificity of the drugs delivered to the tumor cells. Folate is an extensively studied ligand as it is stable, inexpensive and has low immunogenicity (7). Furthermore, the expression of folate receptor (FR) is higher in human cancer cells, including HeLa and MCF-7 cells, than in normal cells $(10,11)$. FA-CS-NPs loaded with anticancer agents produced enhanced intracellular accumulation of therapeutic agents, including doxorubicin and gemcitabine, in FR-positive tumor cells, including HeLa (12), B16F1 and SMMC-722192 skin melanoma cells (13), and COLO357 pancreatic cancer cells (14). However, the use of LZ encapsulated in FA-CS-NPs as a natural MDR reversal agent has not been studied.

The aim of the current study was to develop a novel, cost effective LZ-loaded NPs based drug delivery system to target tumor metastasis and to counter MDR during cancer therapy. 
FA-CS was synthesized by conjugating folate to chitosan via amino-acylation reaction and FA-CS-LZ-NPs were prepared by ionotropic gelation methods. Subsequently, the physical properties and biological activity of FA-CS-LZ-NPs were characterized. In addition, the cancer-targeting specificity of FA-CS-LZ-NPs was determined using MCF-7 (FR-positive) and A549 (FR-negative) cells.

\section{Materials and methods}

Reagents. Chitosan (50 kDa; degree of deacetylation, >90\%), folate, 1-(3-dimethylaminoproply)-3-ethylcarbodiimide hydrochloride (EDC), phosphate buffered saline (PBS, pH 7.4), 3-[4,5-dimethylthiazol-2-yl]-2,5-diphenyltetrazoliumbromide (MTT), and dimethylsulfoxide (DMSO) were purchased from Sigma-Aldrich (Merck KGaA, Darmstadt, Germany). Sodium tripolyphosphate (TPP) was purchased from Kermel Chemical Reagent Co., Ltd., Tianjin, China). LZ (2,3,5,6-tetramethylpyrazine) was purchased from Zelang Pharmaceutical Co., Ltd. (Nanjing, China). Methyl alcohol (chromatographic grade) was purchased from Tedia Company, Inc. (Fairfield, OH, USA).

Cell lines. MCF-7 human breast carcinoma cell line and A549 human lung adenocarcinoma cell line were purchased from Blood Research Administration (Tianjin, China). The cells were cultured in Dulbecco's modified Eagle's medium (DMEM) and supplemented with $10 \%$ fetal bovine serum (both from Gibco; Thermo Fisher Scientific, Inc., Waltham, MA, USA), $100 \mathrm{U} / \mathrm{ml}$ penicillin and $100 \mu \mathrm{g} / \mathrm{ml}$ streptomycin at $37^{\circ} \mathrm{C}$ in a humidified atmosphere with $5 \% \mathrm{CO}_{2}$.

Conjugation and analysis of FA-CS. FA-CS was prepared through an amino-acylation reaction (Fig. 1). Briefly, different concentrations of folate were dissolved into anhydrous DMSO with stirring. EDC $(10 \mathrm{~mol} / \mathrm{ml})$ was added into the solution and stirred at room temperature for $1 \mathrm{~h}$. Subsequently, $5 \mathrm{ml}$ chitosan sodium acetate ( $\mathrm{pH}$ 5.0: w/v 5\%) was added to the solution, stirred at $30^{\circ} \mathrm{C}$ in the dark overnight. The resultant mixture was adjusted to $\mathrm{pH} 9.0$ and then dialyzed against $0.1 \mathrm{M}$ sodium phosphate buffer ( $\mathrm{pH}$ 7.4) for 3 days, followed by dialysis against water for 3 days using a dialysis bag (molecular weight cut-off, $12 \mathrm{kDa}$ ). The mixture was frozen at $-48^{\circ} \mathrm{C}$ for $12 \mathrm{~h}$, and it was subsequently isolated using a FreeZone system (2.5L; Labconco, Kansas City, MO, USA); the synthesized product was obtained following $24 \mathrm{~h}$.

The chemical structure of FA-CS was analyzed by infrared spectroscopy (IR; WGH-30; Gang Dong Technology Co., Ltd., Tianjing, China) and ${ }^{1} \mathrm{H}$ nuclear magnetic resonance (NMR; in $\mathrm{D}_{2} \mathrm{O}, 500 \mathrm{MHz}$; Bruker Corporation, Billerica, MA, USA). The coupled number (the number of folate molecules to CS) of folate to chitosan was calculated based on the molar extinction coefficient value, which was determined by UV-1601 spectrophotometer (Shimadzu Corporation, Kyoto, Japan) at $363 \mathrm{~nm}$.

Preparation and physicochemical characterization of FA-CS-LZ-NPs. FA-CS-LZ-NPs were prepared according to ionotropic gelation method in the following steps. LZ (20 mg) was added to different coupled numbers of FA-CS solution $(2 \mathrm{mg} / \mathrm{ml})$ in $20 \mathrm{ml} \mathrm{1 \%}(\mathrm{w} / \mathrm{w})$ acetic acid $(\mathrm{pH} \mathrm{5.0)}$ at room temperature. TPP $(1 \mathrm{mg} / \mathrm{ml} ; 5 \mathrm{ml})$ was added into the FA-CS solution and stirred using a magnetism mixer $(200 \mathrm{rpm} / \mathrm{min}$; $2,000 \mathrm{x} \mathrm{g}$ ) at room temperature for $1 \mathrm{~h}$. The mixture was then centrifuged at $100,000 \mathrm{x} \mathrm{g}$ for $30 \mathrm{~min}$, the supernatant was collected and the encapsulation efficiency (EE) and loading efficiency (LE) were determined. The precipitate was dispersed in $20 \mathrm{ml}$ deionized water and adjusted $2 \mathrm{mg} / \mathrm{ml}$, and FA-CS-LZ-NPs were isolated by lyophilization. The particle sizes and poly dispersities of FA-CS-LZ-NPs diluted in deionized water were determined using dynamic light scattering system Zetasizer Nano ZS90 (Malvern Instruments, Ltd., Malvern, UK) in triplicate.

FA-CS-LZ-NPs were dissolved with deionized water to $1 \mathrm{mg} / \mathrm{ml}$ and adjusted to neutral $\mathrm{pH}$. One-drop sample was placed on a carbon coated film 300 mesh copper grid and allowed to sit for $5 \mathrm{~min}$ or until air-dried. The sample was stained with $1 \mathrm{M}$ phosphotungstic acetate solution for $5 \mathrm{~min}$, and any excess phosphotungstic acetate was removed with filter paper. Morphological characteristics of the nanoparticles were examined using a high resolution transmission electron microscope (TEM; JEM-2000EX; JEOL, Ltd., Tokyo, Japan).

Determination of EE and LE. The supernatant collected during the final stages of FA-CS-LZ-NPs synthesis as described above was analyzed to determine the amount of free LZ by high performance liquid chromatography (HPLC; programmable solvent module 125; Beckman Coulter, Inc., Brea, CA, USA). The chromatographic conditions used were as follows: HypersilODS- $\mathrm{C}_{18}$ column (250x4.6 mm, $\left.5 \mu \mathrm{m}\right)$; A, methanol; $B$, water (A:B=60:40, v:v, HPLC grade); wavelength, $280 \mathrm{~nm}$; flow rate, $1.0 \mathrm{ml} / \mathrm{min}$; and $25^{\circ} \mathrm{C}$. EE and $\mathrm{LE}$ were calculated as follows: $\mathrm{LZ} \mathrm{EE}(\%)=\left(\mathrm{W}_{\text {totalLZ }}-\mathrm{W}_{\text {free LZ }}\right) / \mathrm{W}_{\text {totalLZ }} \times 100$; and LZ LE $(\%)=\left(\mathrm{W}_{\text {totalLZ }}-\mathrm{W}_{\text {free LZ }}\right) / \mathrm{W}_{\mathrm{NPs}} \times 100 . \mathrm{W}_{\text {totalLZ }}$ was the total amount of added LZ; $W_{\text {free } L Z}$ was the amount of free LZ in the supernatant; and $\mathrm{W}_{\mathrm{NPs}}$ was the weight of FA-CS-LZ-NPs after lyophilization.

In vitro LZ release. FA-CS-LZ-NPs (100 mg) was resuspended in $50 \mathrm{ml}$ deionized water for injection and dialyzed using a membrane dialysis bag (molecular weight cut-off, $12 \mathrm{kDa}$ ) in PBS at pH 5.0, 7.4 and/or 9.0 at $37 \pm 0.5^{\circ} \mathrm{C}$ with continuous stirring. Dialysis buffer $(5 \mathrm{ml})$ was drawn out of the dialysis bag at regular intervals. The concentration of the released LZ in the solutions collected at different time interval was determined by HPLC.

Cytotoxicity assay. MCF-7 (FR-positive) (11) and A549 (FR-negative) cells were seeded at a density of $1 \times 10^{4} /$ well in $100 \mu \mathrm{l}$ culture media in a 96 -well culture plate and incubated for 24 h. Different concentrations $(1,0.5,0.25,0.05$ or $0.01 \mathrm{mg} / \mathrm{ml})$ of FA-CS-LZ-NPs were added to the cells and incubated for $48 \mathrm{~h}$ at $37^{\circ} \mathrm{C}$. MTT solution $(0.5 \%, 10 \mu \mathrm{l})$ was added to the cells in each well and incubated for another $4 \mathrm{~h}$ and $100 \mu \mathrm{l}$ DMSO was added. Absorbance was detected on a microplate reader at $492 \mathrm{~nm}$. The cell viability (\%) was calculated as [optical density (OD) of treated group/OD of control group] x 100.

Intracellular uptake of FA-CS-LZ-NPs. MCF-7 or A549 cells were seeded at a density of $1 \times 10^{4} /$ well in 12 -well culture plate. FA-CS-LZ-NPs, CS-LZ-NPs and LZ solution (the final 


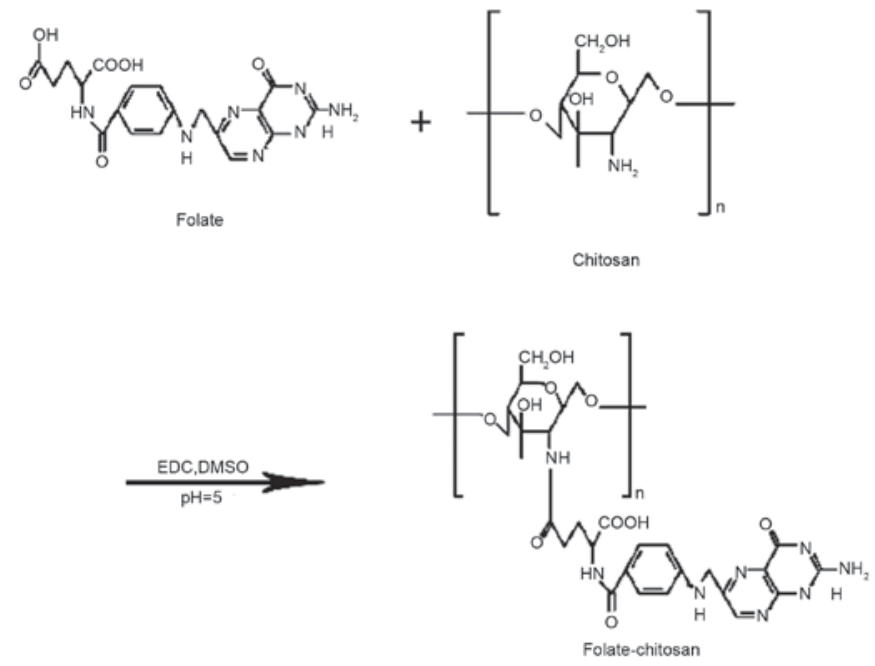

Figure 1. Schematic process of folate-chitosan conjugation. Folate-chitosan is synthesized by the reaction of the activated folate ester with the amine group on the chitosan. EDC, 1-(3-dimethylaminoproply)-3-ethylcarbodiimide hydrochloride; DMSO, dimethyl sulfoxide.

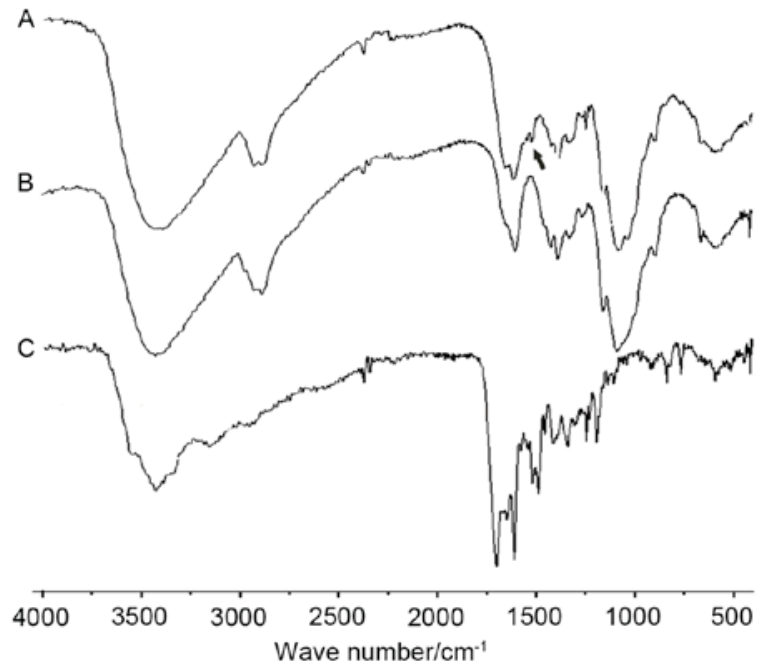

Figure 2. IR spectrum of (A) FA-CS, (B) CS and (C) FA. IR (KBr): $3,372 \mathrm{~cm}^{-1}$ ( $\mathrm{O}-\mathrm{H}, \mathrm{N}-\mathrm{H}$ stretching vibration), 2,924, 2,880 $\mathrm{cm}^{-1}$ (C-H stretching vibration), $1,642,1,600 \mathrm{~cm}^{-1}$ (N-H in-plane bending vibration), $1,420 \mathrm{~cm}^{-1}\left(-\mathrm{CH}_{2},-\mathrm{CH}_{3}\right.$ deformation vibration), $1,560 \mathrm{~cm}^{-1}\left(\mathrm{O}=\mathrm{C}-\mathrm{N}\right.$ stretching vibration), $1,070 \mathrm{~cm}^{-1}$ (O-H deformation vibration), $1,028 \mathrm{~cm}^{-1}$ (C-O stretching vibration), 1,158, $896 \mathrm{~cm}^{-1}$ ( $\beta$-glycosidic bond). FA-CS had a new band at $1,560 \mathrm{~cm}^{-1}$, which stands for amide linkage. FA, folate; CS, chitosan; IR, infrared spectroscopy.

LZ concentration of $50 \mu \mathrm{g} / \mathrm{ml}$ in each group) were added to the wells and cultured for 1, 2, 4, 6 or $8 \mathrm{~h}$. Subsequently, the cells were rinsed with PBS three times, digested with $0.25 \%$ trypsin, and centrifuged at 2,000 x g. Methanol $(100 \mu \mathrm{l})$ was added into cell suspension, the cell mixture was freeze-thawed repeatedly. The LZ concentration of each sample was determined by HPLC.

The autofluorescence intensity of LZ in the cells was monitored by a SP5 laser scanning confocal microscopy (NanoFocus AG, Oberhausen, Germany). The excitation and emission wavelengths of LZ were 295 and $345 \mathrm{~nm}$, respectively. MCF-7 and A549 cells were seeded into $35 \mathrm{~mm}$ cell culture plates at a density of $1 \times 10^{5} /$ plate. The MCF-7 cells were added with $100 \mu 1$ FA-CS-LZ-NPs, CS-LZ-NPs or LZ solution (final LZ concentration, $50 \mu \mathrm{g} / \mathrm{ml}$ in each group) and cultured for $4 \mathrm{~h}$, with A549 cells with $100 \mu 1$ FA-CS-LZ-NPs (final LZ concentration, $50 \mu \mathrm{g} / \mathrm{ml}$ ) as the negative control. The green fluorescence of LZ was monitored at excitation wavelength $295 \mathrm{~nm}$.

FA-CS-LZ-NPs targeting in MCF-7 cells. MCF-7 and A549 cells were cultured in FA-depleted DMEM for 1 week, seeded at a density of $1 \times 10^{5} /$ well in a 12 -well plate and incubated for $24 \mathrm{~h}$. Then, the cells were treated with folate $(0.2 \mu \mathrm{g})$ or untreated. Subsequently, MCF-7 cells were treated with $100 \mu 1$ FA-CS-LZ-NPs, CS-LZ-NPs or LZ solution at $50 \mu \mathrm{g} / \mathrm{ml}$ for $4 \mathrm{~h}$. A549 cells were treated with $100 \mu 1$ FA-CS-LZ-NPs (50 $\mu \mathrm{g} / \mathrm{ml})$ for $4 \mathrm{~h}$. The $\mathrm{LZ}$ concentration for each sample was determined by HPLC.

Statistical analysis. All experiments were repeated at least three times and the data are presented as the mean \pm standard deviation. Tukey's test was performed to determine statistical significance and a $\mathrm{P}<0.05$ was considered to indicate a statistically significant difference.

Ethical statement. The experimental protocol was approved by the Ethics Committee of the Second Hospital of Dalian Medical University (Dalian, China).

\section{Results}

Chemical structure of FA-CS. The functional groups of FA-CS were prepared by an amino-acylation reaction and characterized by infrared spectroscopy. Folate-modified chitosan (Fig. 2A) was contrasted to either folate or chitosan alone (Fig. 2B and C). The basic characteristics of IR bands of chitosan at $3,372 \mathrm{~cm}^{-1}$ was attributed to the stretching vibration of O-H and N-H (Fig. 2B). The strong bands observed at 1642 and $1,600 \mathrm{~cm}^{-1}$ were attributed to the bending vibration of N-H (Fig. 2C). Not only the characteristic bands of the original chitosan but also the characteristic bands of folate were observed by the FA-CS IR spectrum (Fig. 2A). Compared with the chitosan spectrum, a new IR band at $1,560 \mathrm{~cm}^{-1}$, which shows an amide linkage was identified in folate modified chitosan.

The ${ }^{1} \mathrm{H}$ NMR spectrum of FA-CS (Fig. 3A) and chitosan (Fig. 3B) were compared, and observed that the ${ }^{1} \mathrm{H}$ NMR $\left(\mathrm{D}_{2} \mathrm{O}\right) \delta$ spectrum of FA-CS exhibited the new peaks at 2.5 (br s, $\beta$ and $\gamma$-CH2-groups, $4 \mathrm{H}$ ), $7.7 \mathrm{ppm}$ (benzene ring), 6.9 (s, aromatic $\mathrm{H}$ of pteridine) and $8.8(\mathrm{~s}, \mathrm{OH}, 1 \mathrm{H})$ as presented in Fig. 3. The two peaks 4.3 (br s, NH2, 2H) and 4.5 (d, -NH-, $1 \mathrm{H})$ of FA-CS are not vivid in the spectrum because of the high molecular weight of FA-CS (50 kDa). The appearance of these peaks confirmed the successful conjugation of folate with chitosan.

The degree of the substitution of folate on chitosan was calculated by UV spectrophotometry. The folate concentration conjugated with chitosan was directly proportional to the coupled ratio and coupled number (Table I). Coupled ratio of folate and chitosan of 170:1 ( $\mathrm{mol} / \mathrm{mol})$, the number of folate in each chitosan (molecular weight $5 \times 10^{4} \mathrm{Da}$ ), achieved the highest coupled number of 34 . 


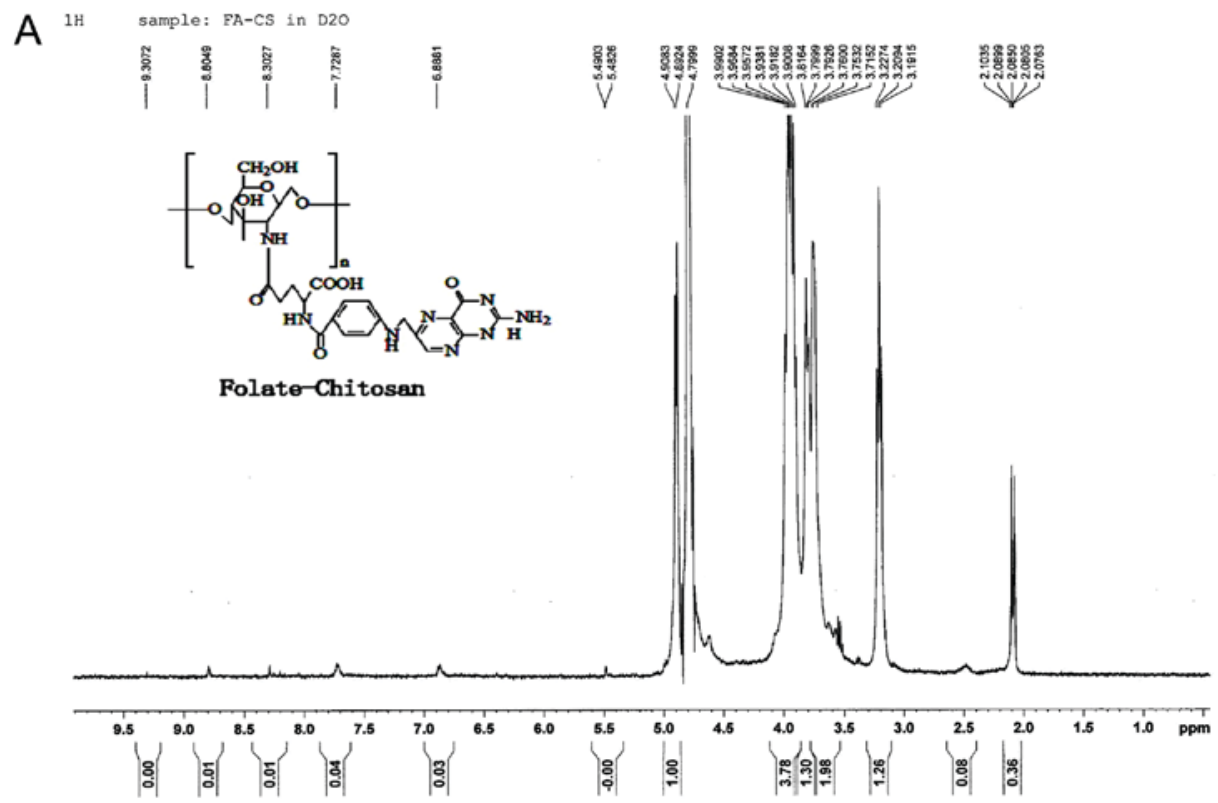

B ${ }^{1 H} \quad$ sample: Cs in D2O

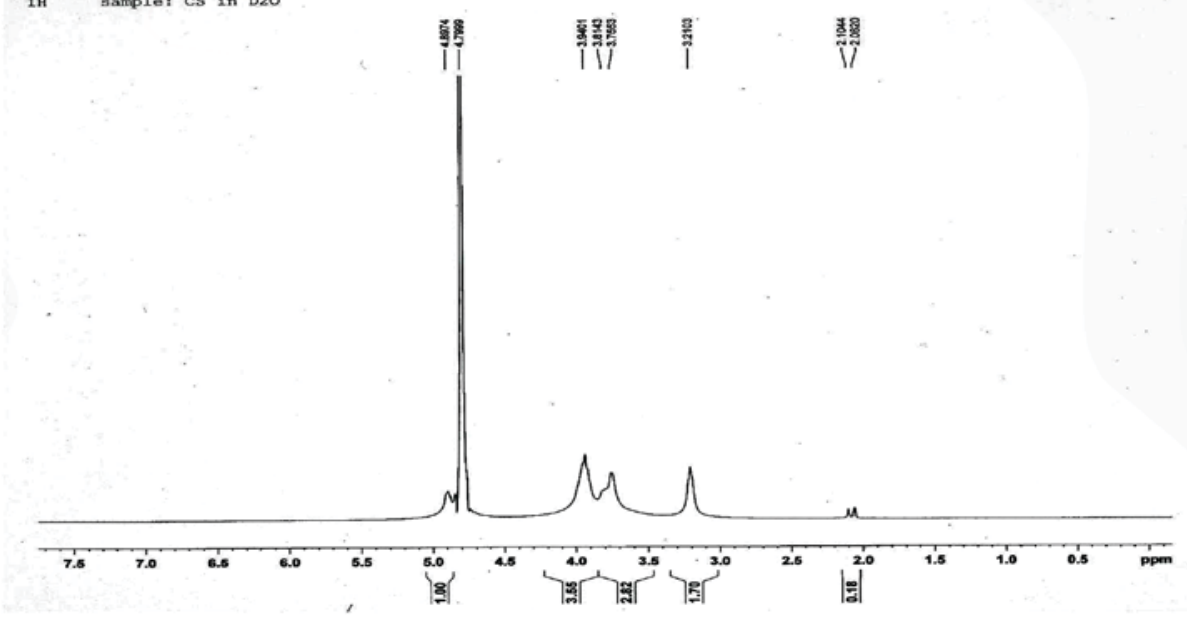

Figure 3. ${ }^{1} \mathrm{H}$ NMR spectrum of (A) folate chitosan and (B) chitosan. ${ }^{1} \mathrm{H}$ NMR $\left(\mathrm{D}_{2} \mathrm{O}, 500 \mathrm{MHz}\right.$ ): $2.5 \mathrm{ppm}$ (br s, $\beta$ and $\gamma$ - $\mathrm{CH}_{2}$-groups, $4 \mathrm{H}$ ), $7.7 \mathrm{ppm}$ (benzene ring), $6.9 \mathrm{ppm}$ (s, aromatic $\mathrm{H}$ of pteridine) and $8.8 \mathrm{ppm}(\mathrm{s}, \mathrm{OH}, 1 \mathrm{H})$. NMR, nuclear magnetic resonance.

Table I. Coupled number of folate on chitosan at different ratios.

\begin{tabular}{cccc}
\hline Folate/mol & Chitosan/mol & $\begin{array}{c}\text { Coupled } \\
\text { ratio }\end{array}$ & $\begin{array}{c}\text { Coupled } \\
\text { number }\end{array}$ \\
\hline 9 & 1 & 0.035 & 4 \\
17 & 1 & 0.1023 & 12 \\
45 & 1 & 0.1936 & 22 \\
56 & 1 & 0.1862 & 21 \\
113 & 1 & 0.2432 & 27 \\
170 & 1 & 0.2929 & 34 \\
\hline
\end{tabular}

With the enhancement of folate concentration the coupled of FA-CS is increasing. Coupled number is the number of folates in each chitosan chain.

Based on the results of IR spectroscopy, ${ }^{1} \mathrm{H}$ NMR spectrums and UV spectrophotometer, it was concluded that folate was conjugated to chitosan.

Physicochemical characterization of FA-CS-LZ-NPs. FA-CS-LZ-NPs were prepared with different coupled number of folate-modified chitosan. The EE, LE and mean diameter size (MD) of FA-CS-LZ-NPs were decreased with the increasing coupled numbers in FA-CS (Table II). The morphology of FA-CS-LZ-NPs was determined by TEM (Fig. 4). FA-CS-LZ-NPs and FA-CS-NPs had uniform spherical morphology, and the distribution of particle size was in the range of 100-200 $\mathrm{nm}$. These results were in agreement with the MDs of the prepared nanoparticles as determined by dynamic light scattering measurements using a Zetasizer Nano ZS90.

In vitro release of $L Z$. The release of $L Z$ from FA-CS-LZ-NPs in PBS at $\mathrm{pH}$ 5.0, 7.4 or 9.0 was investigated. The percentages of LZ released are presented in Fig. 5. The LZ released reached equilibrium at the end of 4 days. The cumulative release proportion was $\sim 95 \%$. The $\mathrm{pH}$ value of dissolution medium 
Table II. EE, LE and MD of different coupled number FA-CS-LZ-NPs $(\mathrm{n}=3)$.

\begin{tabular}{lccc}
\hline Coupled number & EE $(\%)$ & LE $(\%)$ & MD (nm) \\
\hline 4 & $67.4 \pm 0.97$ & $18.1 \pm 0.68$ & $221.8 \pm 1.22$ \\
12 & $63.8 \pm 0.34$ & $16.9 \pm 0.21$ & $194.0 \pm 0.70$ \\
22 & $59.6 \pm 0.23$ & $15.3 \pm 0.16$ & $182.7 \pm 0.56$ \\
34 & $55.7 \pm 0.27$ & $13.8 \pm 0.18$ & $174.9 \pm 0.91$ \\
\hline
\end{tabular}

With the increasing of coupled number the EE, LE and MD are decreased. FA-CS-LZ-NPs, folate-conjugated chitosan ligustrazine nanoparticles; EE, encapsulation efficiency; LE, loading efficiency; $\mathrm{MD}$, mean diameter size.
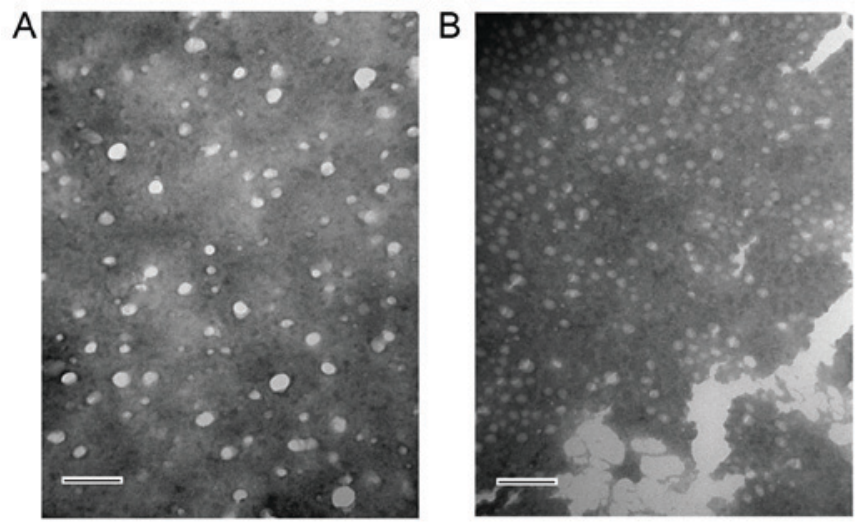

Figure 4. Transmission electronic micrograph of (A) FA-CS-LZ-NPs and (B) FA-CS-NPs. Scale bar, $500 \mathrm{~nm}$. FA-CS-LZ-NPs have uniform spherical morphology. FA-CS-LZ-NPs, folate-conjugated chitosan ligustrazine nanoparticles.

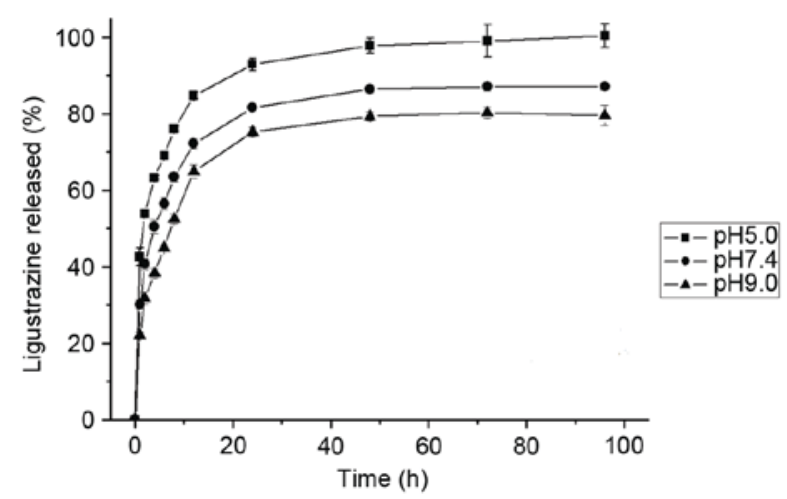

Figure 5. Cumulative release curve of FA-CS-LZ-NPs in PBS at different $\mathrm{pH}$. Data are presented as the mean \pm standard deviation $(n=3)$. The ligustrazine released reaches equilibrium at the end of 4 days. The cumulative release proportion was $\sim 95 \%$. FA-CS-LZ-NPs, folate-conjugated chitosan ligustrazine nanoparticles.

was found to affect the release rate of FA-CS-LZ-NPs. The LZ release rate from the nanoparticles increased with decrease in $\mathrm{pH}$ of dissolution medium, suggesting that $\mathrm{LZ}$ release from FA-CS-LZ-NPs may be higher in in a weak acidic tumor microenvironment.

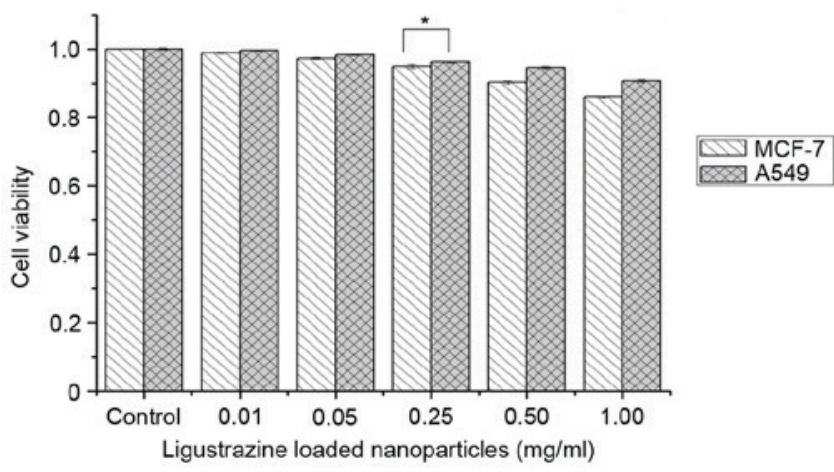

Figure 6. Cytotoxicity of FA-CS-LZ-NPs in MCF-7 and A549 cells. Data are presented the mean \pm standard deviation $(n=3) ;{ }^{*} \mathrm{P}>0.05$. The non-toxic dose of FA-CS-LZ-NPs for MCF-7 cells was $0.25 \mathrm{mg} / \mathrm{ml}$, and the dosage of LZ was $50 \mu \mathrm{g} / \mathrm{ml}$. There was no significant difference between MCF-7 and A549 cells in toxicity. FA-CS-LZ-NPs, folate-conjugated chitosan ligustrazine nanoparticles.

In vitro toxicity assessment. Cytotoxicity of the FA-CS-LZ-NPs in MCF-7 and A549 cells was evaluated using MTT assay. The non-toxic dose was determined as the concentration at which cell viability was $>95 \%$. The non-toxic dose of FA-CS-LZ-NPs in MCF-7 cells was $0.25 \mathrm{mg} / \mathrm{ml}$, and the non-toxic dose of LZ in the two cell types was $50 \mu \mathrm{g} / \mathrm{ml}$. Therefore, these dosages were chosen for all subsequent experiments. The toxicity of FA-CS-LZ-NPs was not significantly different between the cell lines (Fig. 6).

Intracellular uptake of FA-CS-LZ-NPs. MCF-7 and A549 cells were incubated with FA-CS-LZ-NPs, CS-LZ-NPs or $\mathrm{LZ}$ alone solution, at a concentration of $50 \mu \mathrm{g} / \mathrm{ml} \mathrm{LZ}$ in each group. The LZ concentration of each sample was determined by HPLC in at 1, 2, 4, 6, or $8 \mathrm{~h}$ after incubation with MCF-7 and A549 cells. The intracellular concentration of LZ in cells treated with FA-CS-LZ-NPs was significantly higher than cells treated with CS-LZ-NPs or the LZ solution only. The LZ concentration increased between 1 and $4 \mathrm{~h}$, and reached a peak at $6 \mathrm{~h}$ (Fig. 7A); however, no significant difference in the intracellular LZ concentration was observed between the FA-CS-LZ-NP and CS-LZ-NP-treated A549 cells (Fig. 7B). Additionally, at $4 \mathrm{~h}$, the intracellular LZ $(45.47 \pm 0.32 \mu \mathrm{g} / \mathrm{ml})$ in MCF-7 cells treated with the FA-CS-LZ-NPs was significantly higher than that in the A549 cells $(20.10 \pm 0.92 \mu \mathrm{g} / \mathrm{ml})$. These results indicated that FA-CS-LZ-NPs were able to specifically target and deliver LZ into FR-positive MCF-7 cells.

The intracellular uptake efficiency of LZ in MCF-7 cells and A549 cells was also investigated using laser confocal microscopy. Fluorescence intensity of LZ in MCF-7 cells treated with FA-CS-LZ-NPs was higher than that in MCF-7 cells treated with CS-LZ-NPs, LZ solution and the FR-negative A549 cells treated with FA-CS-LZ-NPs, indicating that FA-CS-LZ-NPs facilitated intracellular uptake of LZ (Fig. 8).

FR specific targeting of MCF-7 cells by FA-CS-LZ-NPs. To further explore the mechanism by which FA-CS-LZ-NPs target the tumor cells, a folate competition assay was performed and the intracellular concentration of LZ was determined by HPLC (Fig. 9). Under folate deficient conditions, the intracellular concentration of $\mathrm{LZ}$ in MCF-7 cells 


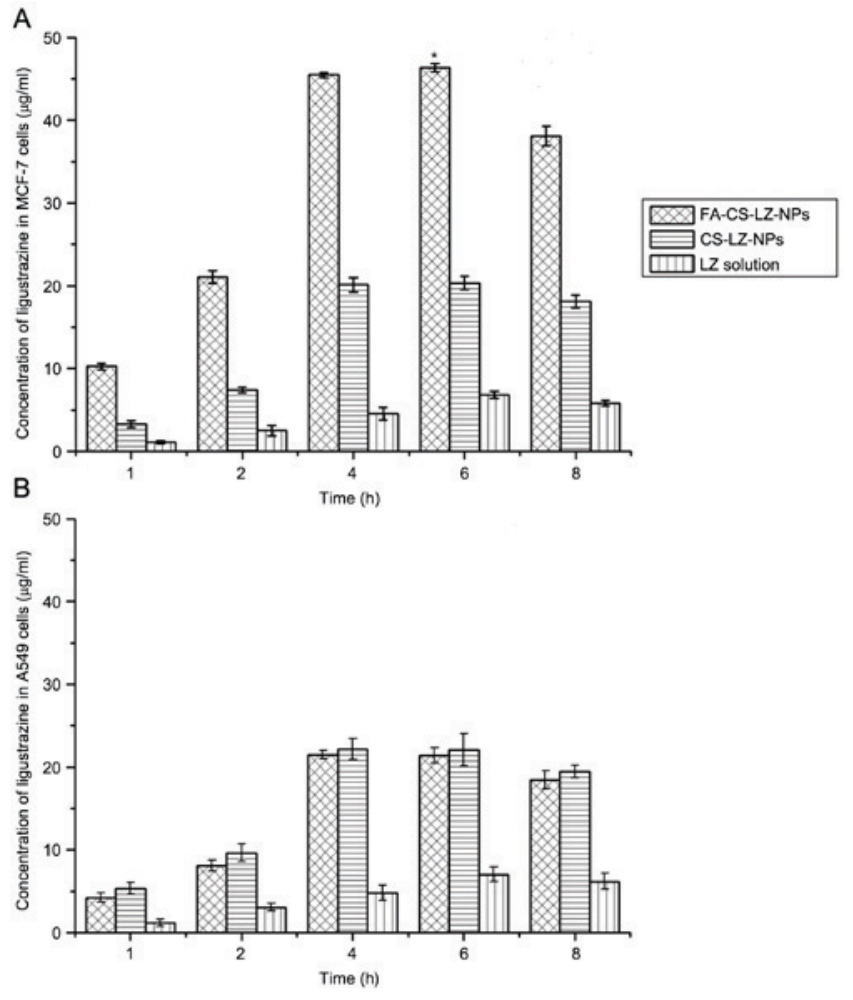

Figure 7. Cellular uptake of ligustrazine at different time points. (A) MCF-7 cells, (B) A549 cells. Data are presented as the mean \pm standard deviation $(\mathrm{n}=3)$. ${ }^{*} \mathrm{P}<0.05$ vs. CS-LZ-NPs. FA-CS-LZ-NPs, folate-conjugated chitosan ligustrazine nanoparticles; CS-LZ-NPs, chitosan ligustrazine nanoparticles.
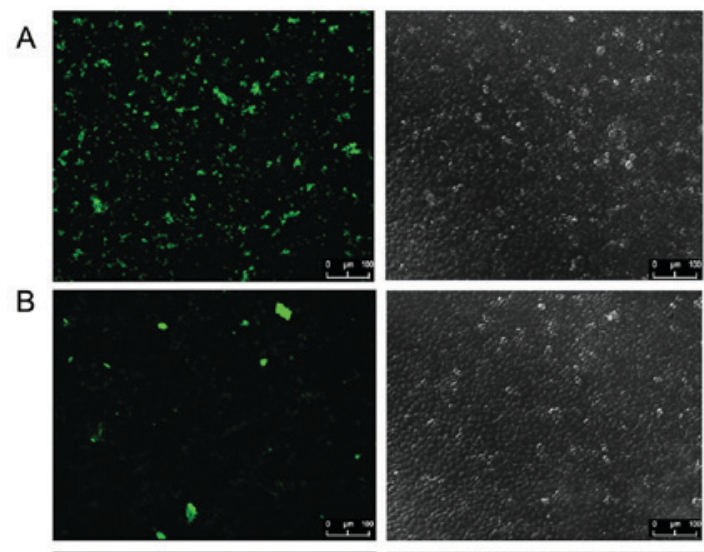

C
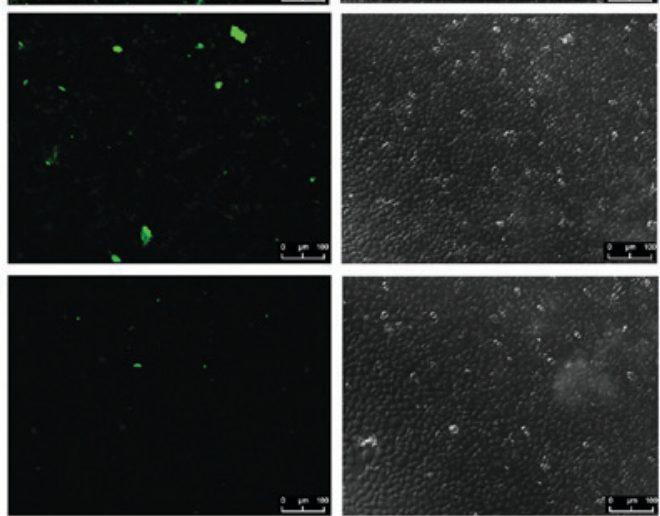

$\mathrm{D}$
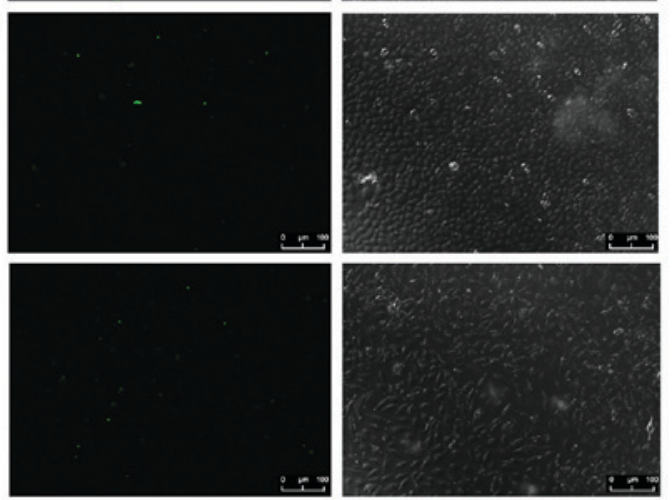

Figure 8. Cellular uptake of ligustrazine in cells after $4 \mathrm{~h}$. A confocal microscope was used to image (A) MCF-7 cells with FA-CS-LZ-NPs, (B) MCF-7 cells with FA-CS-NPs, (C) MCF-7 cells with ligustrazine solution and (D) A549 cells with FA-CS-LZ-NPs. Bar=100 $\mu \mathrm{m}$. FA-CS-LZ-NPs, folate-conjugated chitosan ligustrazine nanoparticles.

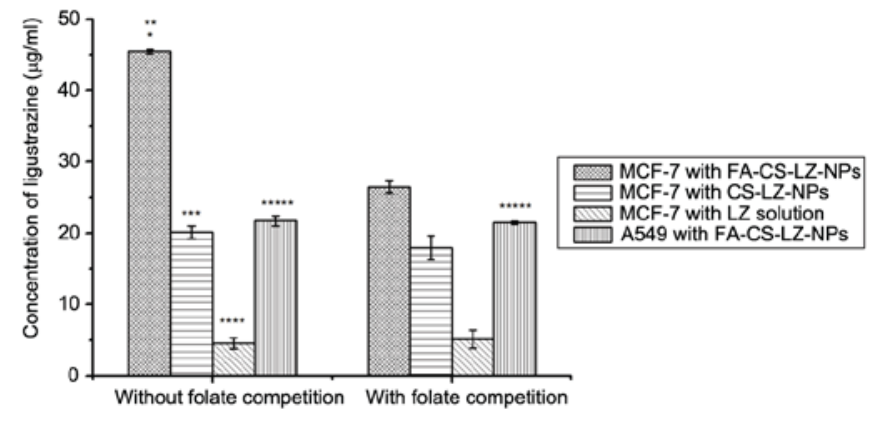

Figure 9. The concentration of ligustrazine in MCF-7 and A549 cells lines. Data are presented as the mean \pm standard deviation $(n=3)$. ${ }^{*} \mathrm{P}<0.01$ vs MCF-7 cells FA-CS-LZ-NPs with folate group; ${ }^{* *} \mathrm{P}<0.01$ vs. other groups without folate competition; ${ }^{* * *} \mathrm{P}<0.05$ vs. MCF-7 cells CS-LZ-NPs with folate group; ${ }^{* * * *} \mathrm{P}>0.05$ compared with MCF-7 cells LZ solution with folate group; ${ }^{* * * * * *} \mathrm{P}>0.05$ compared with A549 cells FA-CS-LZ-NPs with folate group. FA-CS-LZ-NPs, folate-conjugated chitosan ligustrazine nanoparticles.

treated with FA-CS-LZ-NPs was significantly higher than that of the CS-LZ-NPs and LZ solution group. By contrast, the LZ concentration in the MCF-7 cells cultured with folate and treated with FA-CS-LZ-NPs markedly decreased compared to MCF-7 cells with FA-CS-LZ-NPs in folate deficient conditions. However, these differences were not observed in FR-negative A549 cells. Furthermore, addition of folate did not obviously affect the intracellular uptake LZ in cells that were treated with CS-LZ-NPs or LZ solutions. These results suggested that FA-CS-LZ-NPs were internalized via FR.

\section{Discussion}

NPs have been extensively investigated as a delivery system to achieve site-specific delivery of cytotoxic anticancer agents. Chitosan was selected as the nanoparticle carrier material in the present study as its amino groups can conjugate with moieties specific for a cell type or tumor cells (15). Furthermore, chitosan modified with special materials, including folate and arginylglycylaspartic acid polypeptides, functions as an active receptor-mediated targeted drug delivery system. Folate is a stable, inexpensive and poorly immunogenic chemical with a high affinity for FR (16). FA-CS-NPs have been loaded with anticancer agents, such as doxorubicin (17), 5-fluorouracil (18) and used as DNA (19) delivery vehicle, in previous studies. LZ has been reported to have anticoagulation, antioxidation and calcium antagonism properties, thus, is widely used in the management of cardiovascular and cerebrovascular diseases (20). In addition, a number of studies have reported that LZ has an important role in reversing MDR $(2,21)$. In the current study, FA-CS-NPs were used as a vehicle to deliver a natural product (LZ) with the potential to reverse MDR.

In this study, chitosan NPs were modified by folate and loaded with LZ to improve the targeting specificity of NPs to FR-positive cells. The synthesized FA-CS-LZ-NPs had desirable diameter, encapsulation efficiency and uniform spherical morphology. The average size of FA-CS-LZ-NPs with 22 folate-modified chitosan was $182.7 \pm 0.56 \mathrm{~nm}$ and those with 34 folate modified chitosan were $174.9 \pm 0.91 \mathrm{~nm}$. The size and surface charge of nanoparticles could be changed by the concentration ratio of chitosan, folate and TPP. Fewer folate 

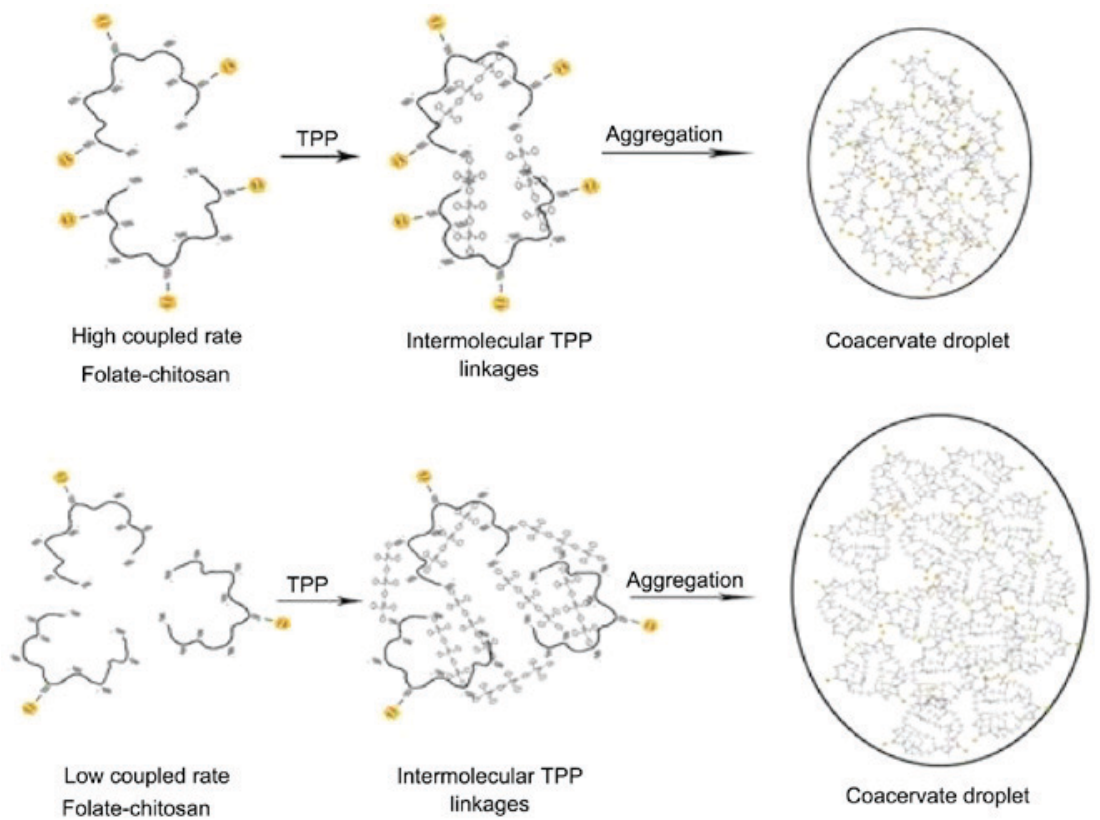

Figure 10. The schematic diagram of high/low coupled rate folate-chitosan to form nanoparticles. TPP, tripolyphosphate.

on one chitosan chain increased the size of nanoparticles because of the free amino groups and low steric hindrance of the folate (Fig. 10). The size of FA-CS-LZ-NPs (182.7 nm) were smaller than folate-modified carboxymethyl chitosan NPs diameter (267.8 nm) (22) and folic acid-conjugated human serum albumin nanoparticles diameter (239 nm) (23). The size of FA-CS-NPs $(182.7 \mathrm{~nm})$ is appropriate for LZ delivery, in contrast with folate-conjugated hyaluronic acid polymeric micelles at size $191.9 \mathrm{~nm}$ are effective in paclitaxel delivery (24). It was observed that $\mathrm{pH}$ affects the release rate of LZ from FA-CS-LZ-NPs. The faster drug release rate at lower $\mathrm{pH}$ may be due to the stronger protonation of amino groups of chitosan which caused the loose nanoparticle structure and higher solubility of $\mathrm{LZ}$ at lower $\mathrm{pH}$. The $\mathrm{LZ}$ release rate from the nanoparticles increased with a decrease in $\mathrm{pH}(5.0)$ of the dissolution medium, suggesting that $\mathrm{LZ}$ release from FA-CS-LZ-NPs may be higher in a weak acidic microenvironment. As the tumor microenvironment is slightly acidic (25), FA-CS-LZ-NPs may have potential as an anticancer system. The cell viability profile assessed by MTT assay demonstrated that FA-CS-NPs had no significant cytotoxicity at concentrations as high as $1 \mathrm{mg} / \mathrm{ml}$, so FA-CS-LZ-NPs may be a safe tool that could potentially overcome MDR during cancer therapy. FA-CS-NPs targeted MCF-7 cells via FR without obvious cytotoxicity. In fact, chitosan NPs were demonstrated to be a suitable and controlled drug delivery system without significant cytotoxicity in liver cells (26), and folate gold nanoparticles do not induce significant cytotoxicity in MCF-7 cells (27). In this study, internalized FA-CS-LZ-NPs detected in MCF-7 cells cultured with folate may be attributed to the fact that folate at $0.2 \mu \mathrm{g} / \mathrm{ml}$ occupied all the folate receptors on the cells. The intracellular concentration of LZ in MCF-7 cells following exposure to FA-CS-LZ-NPs was significantly higher than that of cells exposed to CS-LZ-NPs and LZ alone. Folate and FR may be the main cause of FA-CS-LZ-NP cellular uptake. FA-CS-NPs may be a more suitable vehicle, compared with
CS-NPs, for overcoming tumor drug resistance due to its high cellular uptake specificity by FR-expressing cells.

In conclusion, the FA-CS-LZ-NPs developed in the current study had suitable diameter, high EE and LE, uniform spherical morphology, and slow release in vitro, but no cytotoxicity. FA-CS-LZ-NPs may be a potential tool to target FR-positive cancer cells and thus, a promising candidate for overcoming MDR.

\section{Acknowledgements}

This work was funded by the Science and Technology Planning Project of Liaoning Province (grant no. 2012225020).

\section{References}

1. Ji XX, Song XL, Qian W, Yu XL and Zhu JY: Effects and mechanism of action of ligustrazine on isoprenaline-induced cardiomyocyte hypertrophy. Cell Biochem Biophys 70: 1513-1518, 2014.

2. Chen J, Wang W, Wang H, Liu X and Guo X: Combination treatment of ligustrazine piperazine derivate DLJ14 and adriamycin inhibits progression of resistant breast cancer through inhibition of the EGFR/PI3K/Akt survival pathway and induction of apoptosis. Drug Discov Ther 8: 33-41, 2014.

3. Xu K, Wang P, Xu X, Chu F, Lin J, Zhang Y and Lei H: An overview on structural modifications of ligustrazine and biological evaluation of its synthetic derivatives. Res Chem Intermed 41: 1385-1411, 2015.

4. Fan Q, Fan GJ, Zhao JY and Yang PM: Study on effect reversing MDR of ligustrazine liposomes on human erythroleukemia-cell-line K562/ADM. China Pharmacist 7: 753-755, 2004.

5. Fan GJ, Fan Q and Leng DW: Preparation and quality evaluation of ligustrazine liposomes. Pharm Care Res 8: 453-455, 2008.

6. Yao Q, Liu W, Gou XJ, Guo XQ, Yan J, Song Q, Chen FZ, Zhao Q, Chen $\mathrm{C}$ and Chen T: Preparation, characterization, and cytotoxicity of various chitosan nanoparticles. J Nanomater 2013: 14, 2013.

7. Li P, Wang Y, Zeng F, Chen L, Peng Z and Kong LX: Synthesis and characterization of folate conjugated chitosan and cellular uptake of its nanoparticles in HT-29 cells. Carbohydr Res 346: 801-806, 2011. 
8. Milane L, Duan ZF and Amiji M: Pharmacokinetics and biodistribution of lonidamine/paclitaxel loaded, EGFR-targeted nanoparticles in an orthotopic animal model of multi-drug resistant breast cancer. Nanomedicine 7: 435-444, 2011.

9. Danhier F, Vroman B, Lecouturier N, Crokart N, Pourcelle V, Freichels H, Jérôme $\mathrm{C}$, Marchand-Brynaert J, Feron $\mathrm{O}$ and Préat V: Targeting of tumor endothelium by RGD-grafted PLGA-nanoparticles loaded with paclitaxel. J Control Release 140: 166-173, 2009.

10. Shen Z, Li Y, Kohama K, Oneill B and Bi J: Improved drug targeting of cancer cells by utilizing actively targetable folic acid-conjugated albumin nanospheres. Pharmacol Res 63: 51-58, 2011.

11. Wang H, Zhao P, Liang X, Gong X, Song T, Niu R and Chang J: Folate-PEG coated cationic modified chitosan-cholesterol liposomes for tumor-targeted drug delivery. Biomaterials 31: 4129-4138, 2010.

12. Shi Z, Guo R, Li W, Zhang Y, Xue W, Tang Y and Zhang Y: Nanoparticles of deoxycholic acid, polyethylene glycol and folic acid-modified chitosan for targeted delivery of doxorubicin. J Mater Sci Mater Med 25: 723-731, 2014.

13. Song H, Su C, Cui W, Zhu B, Liu L, Chen Z and Zhao L: Folic acid-chitosan conjugated nanoparticles for improving tumor-targeted drug delivery. Biomed Res Int 2013: 723158, 2013.

14. Zhou J, Wang J, Xu Q, Xu S, Wen J, Yu Z and Yang D: Folate-chitosan-gemcitabine core-shell nanoparticles targeted to pancreatic cancer. Chin J Cancer Res 25: 527-535, 2013.

15. Nagpal K, Singh SK and Mishra DN: Chitosan nanoparticles: A promising system in novel drug delivery. Chem Pharm Bull (Tokyo) 58: 1423-1430, 2010.

16. Hou Z, Zhan C, Jiang Q, Hu Q, Li L, Chang D, Yang X, Wang Y, Li Y, Ye S, et al: Both FA- and mPEG-conjugated chitosan nanoparticles for targeted cellular uptake and enhanced tumor tissue distribution. Nanoscale Res Lett 6: 563, 2011.

17. Lee KD, Choi SH, Kim DH, Lee HY and Choi KC: Self-organized nanoparticles based on chitosan-folic acid and dextran succinate-doxorubicin conjugates for drug targeting. Arch Pharm Res 37: 1546-1553, 2014.

18. Li HL, He YX, Gao QH and Wu GZ: Folate-polyethylene glycol conjugated carboxymethyl chitosan for tumor-targeted delivery of 5-fluorouracil. Mol Med Rep 9: 786-792, 2014.
19. Mansouri S, Cuie Y, Winnik F, Shi Q, Lavigne P, Benderdour M, Beaumont E and Fernandes JC: Characterization of folate-chitosan-DNA nanoparticles for gene therapy. Biomaterials 27: 2060-2065, 2006.

20. Chen L, Lu Y, Wu JM, Xu B, Zhang LJ, Gao M, Zheng SZ, Wang AY, Zhang CB, Zhang WW and Lei N: Ligustrazine inhibits B16F10 melanoma metastasis and suppresses angiogenesis induced by vascular endothelial growth factor. Biochem Biophys Res Commun 386: 374-379, 2009.

21. Yang XG and Jiang C: Ligustrazine as a salvage agent for patients with relapsed or refractory non-Hodgkin's lymphoma. Chin Med J (Engl) 123: 3206-3211, 2010.

22. Tan YL and Liu CG: Preparation and characterization of self-assembled nanoparticles based on folic acid modified carboxymethyl chitosan. J Mater Sci Mater Med 22: 1213-1220, 2011.

23. Ulbrich K, Michaelis M, Rothweiler F, Knobloch T, Sithisarn P, Cinatl $\mathbf{J}$ and Kreuter J: Interaction of folate-conjugated human serum albumin (HSA) nanoparticles with tumour cells. Int J Pharm 406: 128-134, 2011.

24. Liu Y, Sun J, Cao W, Yang J, Lian H, Li X, Sun Y, Wang Y, Wang S and He Z: Dual targeting folate-conjugated hyaluronic acid polymeric micelles for paclitaxel delivery. Int J Pharm 421: 160-169, 2011.

25. Du J, Lane LA and Nie S: Stimuli-responsive nanoparticles for targeting the tumor microenvironment. J Control Release 219: 205-214, 2015.

26. Loh JW, Yeoh G, Saunders M and Lim LY: Uptake and cytotoxicity of chitosan nanoparticles in human liver cells. Toxicol Appl Pharmacol 249: 148-157, 2010.

27. Shakeri-Zadeh A, Mansoori GA, Hashemian AR, Eshghi H, Sazgarnia A and Montazerabadi AR: Cancerous cells targeting and destruction using folate conjugated gold nanoparticles. Dyn Biochem Process Biotechnol Mol Biol 4: 6-12, 2010. 